\title{
Formação e qualificação das equipes do serviço aeromédico no Brasil
}

RESUMO | Objetivo: Descrever sobre a formação e qualificação dos profissionais de saúde que realizam remoções aéreas, suas particularidades e legislações pertinentes, no Brasil. Método: Trata-se de uma pesquisa descritiva exploratória, sobre o processo de formação, qualificação e atuação profissional no serviço de transporte aéreo. O desenvolvimento do estudo deuse no período de julho a agosto de 2021. Resultado: $O$ transporte aeromédico possui duas áreas com características muito distintas, o setor de aviação e o da medicina aeroespacial. Entretanto, possuem processos muito similares, as regulamentações são fragmentadas, as legislações são inespecíficas e há pouca oferta de formação e/ou capacitações. Assim, é essencial o uso de novas ferramentas para gestão do conhecimento. Conclusão: O processo de formação profissional demanda tempo, qualificação específica e desenvolvimento de habilidades avançadas. Para tal, as equipes devem ser especializadas, experientes, capazes de tomar decisões precisas e rápidas.

Descritores: Enfermeiros; Gestão do conhecimento; Resgate Aéreo; Capacitação; Qualificação.

ABSTRACT | Objective: To disclose about a training and qualification of two health professionals who carry out air removals, their particularities and pertinent legislation, not Brazil. Method: It deals with an exploratory descriptive research, on the process of training, qualification and professional performance not air transport service. Either development of the study from July to August 2021. Result: Either aeromedical transport has two areas with very different characteristics, or aviation and aerospace medicine setting. In the meantime, there are very similar processes, the regulations are fragmented, the laws are unspecific and there is little offer of training and / or qualifications. Also, it is essential or use of nova ferramentas for conhecimento management. Conclusion: The professional training process demands time, specific qualification and development of advanced skills. For this, teams must be specialized, experienced, capable of making precise and fast decisions.

Descriptors: Diseases; Gestão do conhecimento; Air Resgate; Training; Qualification.

RESUMEN | Objetivo: Describir la formación y calificación de los profesionales de la salud que realizan remociones aéreas, sus particularidades y legislación relevante en Brasil. Método: Se trata de una investigación descriptiva exploratoria sobre el proceso de formación, calificación y desempeño profesional en el servicio de transporte aéreo. El desarrollo del estudio se llevó a cabo de julio a agosto de 2021. Resultado: El transporte aeromédico tiene dos áreas con características muy diferentes, el sector de la aviación y el sector de la medicina aeroespacial. Sin embargo, tienen procesos muy similares, la normativa está fragmentada, la legislación es poco específica y hay poca oferta de formación y / o formación. Por tanto, el uso de nuevas herramientas de gestión del conocimiento es fundamental. Conclusión: El proceso de formación profesional demanda tiempo, cualificación específica y desarrollo de competencias avanzadas. Para ello, como equipos, deben ser especializados, experimentados, capaces de tomar decisiones acertadas y rápidas.

Descriptores: Enfermeras; Conocimiento administrativo; Rescate aéreo; Capacitación; Calificación.

Bruno Gonçalves da Silva

Enfermeiro (UNIFENAS), Mestre em Administração de Empresas (Centro Universitário UNA), Doutorando em Sistemas de Informações e Gestão do Conhecimento (Universidade FUMEC). Professor Assistente FCMMG e Enfermeiro de Bordo da Unimed Aeromédica

ORCID: 0000- 0001-5173-0036

\section{Vânia Paula de Carvalho}

Enfermeira (PUC-MINAS), Enfermeira Intensivista (IEC-PUC MG) e Enfermeira Aeroespacial (FIC-UNIVIRTUAL), Mestre em Promoção da Saúde e Prevenção da Violência (UFMG). Coordenadora de Enfermagem/RT da Unimed Aeromédica.

ORCID: 0000-0002-9336-3606

\section{Maria Eduarda Becho Arger Marchetti}

Médica (UFMG), Especialista em Anestesiologista e Medicina Aeroespacial. Médica Emergencista e Horizontal na UPA-CS BH, Anestesista em HCMG e Médica de Bordo na Unimed Aeromédica.

ORCID: 0000-0002-0242-243X

\section{André Alves Elias}

Médico (UNIFENAS). Médico de Bordo na Unimed Aeromédica e BOA-SAMU BH. ORCID:0000-0002- 1103-6450

\section{Flávio Lopes Ferreira}

Médico (UFMG), Mestre em Fisiologia e Farmacologia (UFMG), Cirurgião Geral e Especialista em Medicina Aeroespacial. Gestor Médico da Unimed Aeromédica, Professor na Faculdade de Ciências Médi- cas de Minas Gerais

ORCID: 0000-0001-7740-4394

\section{Armando Sérgio de Aguiar Filho}

Comunicador Social, Professor Titular na Universidade FUMEC e da Faculdade Promove. Doutor em Gestão da Informação e do Conhecimento (UFMG).

ORCID: 0000-0001-5542-7165

Recebido em: 22/09/2021

Aprovado em: 08/10/2021 
INTRODUÇÃO

$\mathrm{N}$ o Brasil, os primeiros registros acerca dos serviços de remoção aeromédica datam de 1.988, pelo Corpo de Bombeiros do Rio de Janeiro associado à Coordenadoria Geral de Operações Aéreas do Estado destinando-se aos resgates, salvamentos e eventualmente remoções secundárias. Em contrapartida, no mercado há instituições particulares que oferecem o serviço e os treinamentos específicos para os profissionais, sob a supervisão do Departamento Civil de Aviação. ${ }^{1}$

A sofisticação crescente do Serviço de Remoção Aeromédica no mundo atende as diferentes necessidades e características de cada país. As principais distinções são estabelecidas a partir das dimensões territoriais, da distribuição heterogênea de recursos médicos, da existência de comunidades isoladas e do número elevado de acidentes de trânsito. $^{2}$

O transporte aéreo de pacientes críticos está na vanguarda do mundo, em constante mudança para adaptação aos tempos modernos. Impulsionado pela demanda do mercado às práticas avançadas de cuidado ao paciente criticamente enfermo, cresce exponenciamente. $^{3}$

A expansão dos provedores de serviços de emergência para o transporte de cuidados intensivos traz consigo uma necessidade premente de educação formal, preparação clínica multifacetada, visão de logística do transporte e expertise em cuidados intensivos. Assim como, poderá contribuir para preencher as lacunas do conhecimento para os profissionais no ambiente aeroespacial. ${ }^{3}$

Define-se pelo transporte por via aérea de feridos ou enfermos, em um ambiente similar a Unidade de Terapia Intensiva, que necessita da atenção de uma equipe altamente especializada, requer uma atenção prioritária e pode ser um determinante importante em seu prognóstico. ${ }^{4}$ Conta com profissionais de práticas avançadas, especialistas experientes, conhecedores de medicina aeroespacial e que consigam se adaptar ao trabalho em ambiente confinado.

No ambiente aéreo, o domínio dos conhecimentos das práticas avançadas pelas equipes multidisciplinares é imprescindível. Faz-se necessário interfaces intersetoriais bem estabelecidas e a capacitação da equipe envolvida, para que o atendimento ao paciente criticamente enfermo seja seguro e de qualidade. $^{5,6}$

O cuidado intensivo é baseado em um tripé: paciente crítico, equipamentos altamente técnicos e equipe multiprofissional especializada. Sendo que, esses fatores são interligados, interdependentes e os recursos humanos treinados minimiza o risco de ocorrências de eventos adversos. ${ }^{7}$

Neste sentido, a evolução dos cuidados com os pacientes transportados, os tipos de ambulâncias utilizadas, as equipes com práticas avançadas, a importância do treinamento efetivo dos mesmos, domínio dos embasamentos sobre medicina aeroespacial, podem corroborar para a segurança e qualidade do transporte. ${ }^{8}$

Assim, o objetivo deste trabalho foi descrever sobre a formação e qualificação dos profissionais de saúde que realizam aerorremoções de pacientes, suas particularidades e legislações pertinentes, no Brasil.

\section{METODOLOGIA}

Tipo de estudo

Trata-se de uma pesquisa descritiva exploratória, sobre o processo de formação, qualificação e atuação do enfermeiro de bordo no serviço de transporte aéreo, no Brasil.

A pesquisa descritiva expõe as características da população específica ou do fenômeno a ser estudado, define sua natureza e faz correlação entre as suas variáveis. ${ }^{9}$ E a pesquisa explora- 
tória visa proporcionar um aprofundamento no problema proposto a fim de possibilitar seu entendimento de forma clara.

Para tal, foi realizada uma pesquisa na Biblioteca Virtual de Saúde com foco no tema proposto, houve uma escassez de publicações, as encontradas apenas citavam a importância da formação e qualificação. A busca deu-se no período de julho a agosto de 2021. Não houve necessidade de apreciação do Comitê de Ética em Pesquisa, pois o estudo não envolveu seres humanos. O registro de imagem foi realizado durante simulação realística de transporte neonatal, pelos autores.

\section{RESULTADOS/DISCUSSÃO}

No Brasil, a atividade de transporte aéreo de enfermos é recente e existem

poucas instituições de ensino que ofertam especialização em enfermagem e/ou em medicina aeroespacial. Ainda, as regulamentações são fragmentadas por formação profissional e as legislações são inespecíficas. ${ }^{10}$

Portanto, a tripulação de saúde deve seguir as normatizações, regulamentações, domínio das particularidades da aeronave e da assistência ao paciente crítico. Neste tipo de transporte, os profissionais devem possuir grande expertise e capacitação para atendimento no ambiente adaptado. ${ }^{11}$

Observa-se que o transporte aeromédico possui duas áreas com características muito distintas, o setor de aviação (piloto e copiloto) e o da medicina aeroespacial (médico e o enfermeiro). Entretanto, possuem processos muito similares quando avaliamos a necessidade de trabalho em equipe, excelente gestão de processos, organização, planejamento, utilização de diretrizes operacionais rígidas, uso de tecnologias de ponta, preocupação com a segurança e altos padrões de qualidade.

O serviço de Transporte Aéreo o qual está inserido no sistema de atendi- mento médico pré-hospitalar de urgência e emergência, passou a ser regulamentado pelas Portarias do Ministério da Saúde GM/MS n 2048 de 05 de novembro de 2002 e no $1.863 / \mathrm{GM}$ de 29 de setembro de 2003, em todo o território nacional., ${ }^{6,12} \mathrm{Na}$ evolução cronológica, para a enfermagem aeroespacial, o Conselho Federal de Enfermagem (COFEN) publicou a Resolução de n 551, de 12 de julho de 2017 , normatizou a atuação do enfermeiro no atendimento pré-hospitalar (APH) móvel e inter-hospitalar em veículo aéreo. Seguida pela Resolução COFEN $n^{\circ}$ 660/2021, de 03 de março de 2021, alterou a Resolução COFEN n ${ }^{\circ} 656$, de 17 de dezembro de 2020, que normatizou a atuação na assistência direta e a gerência do APH e Inter-hospitalar em veículo aéreo. ${ }^{6,12,13}$

Vale lembrar que, durante o translado do paciente crítico a equipe de saúde é composta por dois profissionais, que devem possuir domínio teórico-prático, conhecimentos amplos de medicina aeroespacial, capacidade física e outros. Sendo que, o primordial é desenvolver a capacidade de trabalho em equipe e, consequentemente, perfeita sintonia com o seu parceiro.

A experiência de cada profissional é muito importante, pois em ambiente confinado contamos, exclusivamente, com a expertise do parceiro e esses saberes devem se complementar harmoniosamente. Assim como, a interação entre a equipe deve ser absoluta, sincrônica, com respeito mútuo, comunicação respeitosa, tendo como foco o atendimento qualificado e humanizado ao paciente aerorremovido.

Para isso, o investimento pelas empresas em programas de treinamento com metodologias ativas é primordial. Assim como, a possibilidade do uso de novas ferramentas de ensino.

Já no processo de trabalho assistencial, além das competências do enfermeiro descritas pelo código de ética de enfermagem através da Resolução COFEN 311/2007, a prática da enfermagem de bordo é amparada pela Lei $n^{\circ} 7.498 / 86$, que regulamenta o Exercício Profissional de Enfermagem. Nela, é estabelecido que é privativo do enfermeiro a organização e direção da assistência direta ao paciente crítico e onde sejam executadas atividades de maior complexidade técnica. ${ }^{6,8}$

Existe uma gama de fatores a serem gerenciados e os profissionais devem estar atentos a toda logística e etapas da aerorremoção. Alinhados a formação avançada, conhecimentos técnicos científicos atualizados, ampla experiência, com uma boa dinâmica entre as equipes de voo e de saúde, capacidade

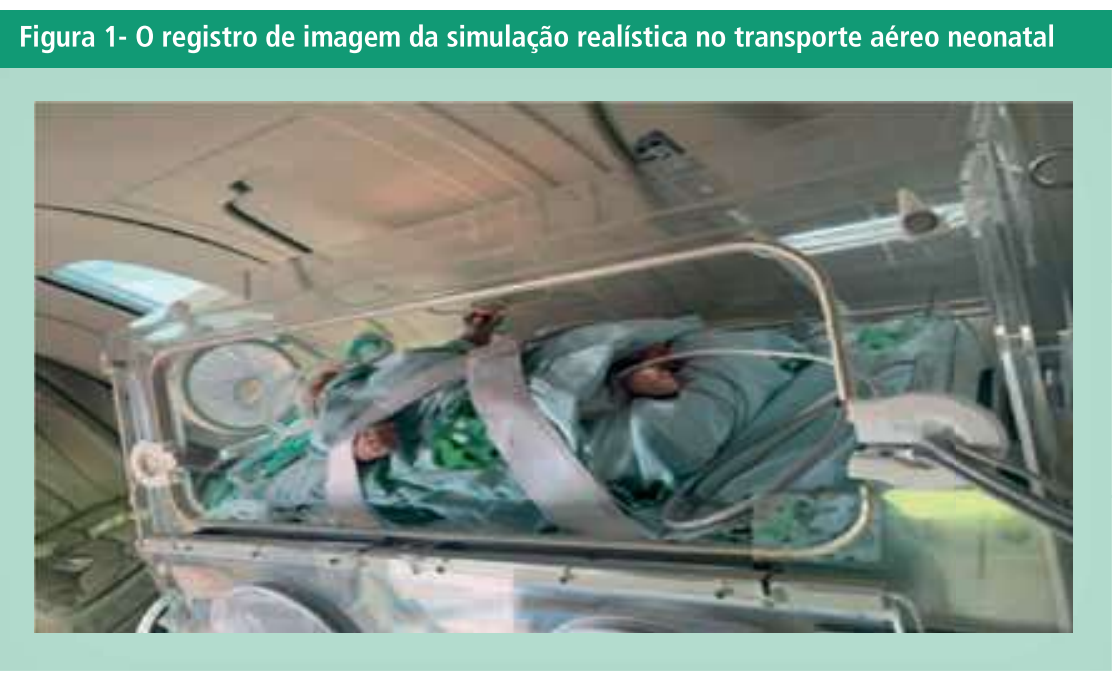

Fonte: Arquivo pessoal dos autores, 2021 
de manuseio de equipamentos e tecnologias necessárias para cuidado durante todo o transporte.

A Resolução COFEN 0551/17 que normatiza a atuação do enfermeiro no atendimento Pré Hospitalar Móvel e Inter-Hospitalar em Veículo Aéreo, que esclarece que o enfermeiro deverá possuir especialização e/ou residência e/ou título de especialista emitido por sociedade ou já estar em exercício na área até a publicação da resolução. ${ }^{6,13}$

Para direcionar o profissional na constituição gerencial dentro de um serviço de resgate aeromédico há a Resolução CFM no 1.671/03, que define os insumos necessários para a ambulância de suporte avançado e os descreve, com as adaptações necessárias para o uso em ambientes hipobáricos. ${ }^{14}$

Ressalta-se a importância dos profissionais conhecerem as legislações que os regulamentam, colaborar para a construção de novas perspectivas na assistência e gerenciamento do paciente crítico em ambiente aéreo. Ainda, o processo de formação e qualificação da equipe de voo demanda tempo e preparo específico extenso. Para isso, os profissionais devem completar a graduação, especialização e/ou realizar residência e manter-se atualizado ao longo de sua carreira profissional. ${ }^{15}$

Neste contexto, o transporte aeromédico deve acontecer quando o benefício de cuidados especiais sobrepõe os riscos do transporte. Considera-se a escolha do tipo de aeronave, a distância do aeródromo, o tempo de translado, as condições de tráfego e meteorológicas, a segurança, a patologia do paciente, a necessidade de intervenções e dentre outros. ${ }^{16}$

Atualmente, um dos principais objetivos dos estudos e trabalhos em saúde é assegurar ao paciente atendimento de qualidade, ofertar melhor assistência, diagnóstico e terapêutica. Para tal, houve mudanças na organização das estruturas hospitalares, tornando-as autossuficientes, especializadas e estrati- ficadas de acordo com a sua complexidade. Para isso, os recursos alocados foram otimizados, assim como, os custos e a rentabilidade. ${ }^{17}$

Ressalta-se que o trabalho em equipe é uma das habilidades indispensáveis para que o transporte transcorra de forma segura e qualificada.

Assim como, programas de treinamento são primordiais, a possibilidade

\section{Ressalta-se a importância dos profissionais conhecerem as legislações que os regulamentam, colaborar para a construção de novas perspectivas na assistência e gerenciamento do paciente crítico em ambiente aéreo.}

do uso de novas ferramentas de ensino como as simulações realísticas e/ou uso de tecnologias. Estes investimentos, alinhados a docentes atualizados poderão corroborar para melhorar a prestação de serviços, a gestão dos sistemas de segurança, qualidade e o trabalho em equipe.

\section{CONCLUSÃO}

A enfermagem e a medicina aeroespacial são áreas de atuação em expansão, o processo de formação de novos profissionais demanda tempo, qualificação específica e desenvolvimento de habilidades avançadas. Portanto, o transporte aéreo de pacientes críticos deve ser realizado por equipes especializadas, experientes, com a capacidade de desenvolver a comunicação de alto nível, capazes de tomar decisões precisas e rápidas.

É premente a necessidade de regulamentações que protejam os profissionais de saúde, reconheçam os profissionais veteranos como especialistas, fiscalizem as prestadoras de serviços de maneira efetiva e invistam na segurança aeroportuária em nosso país. Pela sua singularidade, o processo de formação dos profissionais de saúde é extenso, oneroso, fragmentado e sem regulamentações específicas.

Fica evidente a importância da qualificação, com a finalidade de reconhecer efetivamente as particularidades do atendimento no ambiente aeroespacial e consequentemente, aprimorar a qualidade e segurança do atendimento no transporte aeromédico.

Sobremodo, a atuação do enfermeiro de bordo é multifacetada. Faz-se necessário o empoderar, com intuito de melhorar a práxis e consequentemente, o cuidado holístico e humanizado. Ademais, evidencia-se a importância de novos estudos e o investimento em novas ferramentas para gestão do conhecimento. 


\begin{tabular}{|c|c|c|}
\hline & $\mathbf{R e}$ & cias \\
\hline & $\begin{array}{l}\text { 1- Rocha PK, et al. Assistência de enfermagem em serviço pré-hospitalar e } \\
\text { remoção aeromédica. Rev. Brasileira, de Enferm. [base de dados da Internet]. }\end{array}$ & $\begin{array}{l}\text { polytraumatized patients: review of literature. Rev Cient Sena Aires. [Inter- } \\
\text { net]. } 2016 \text { [cited } 2019 \text { Mar } 21 \text { ]; } 5 \text { (2): 171-77. }\end{array}$ \\
\hline & $\begin{array}{l}\text { 2003. Brasília: [acesso em: } 17 \text { Set 2018]. Disponível em: http://www.sielo.br/ } \\
\text { pdf/rebeu/v56n6/a22v.56n6.pdf. }\end{array}$ & $\begin{array}{l}\text { 11- Thomaz RR, Miranda MFB, Souza GAG, Gentil RC. Enfermeiro de bordo: } \\
\text { uma profissão no ar. Acta paul. enferm.1999;12(1):86-96. }\end{array}$ \\
\hline & $\begin{array}{l}\text { 2- Gentil RC. Aspectos históricos e organizacionais da remoção aeromédica: } \\
\text { A dinâmica da assistência de enfermagem. Rev. Escola de Enfermagem USP. } \\
\text { [base de dados da Internet]. 1997. Brasília: [acesso em: } 25 \text { Ago 2018]. Dispo- } \\
\text { nível em:http://www.scielo.br/pdf/reusp/v31n3/v31/n3a08.pdf } \\
\text { 3- Pollack AN, Murphy M, Stathers LC, Pecora D, McEvoy M, Rabrich JS. Cri- }\end{array}$ & $\begin{array}{l}\text { 12- Gomes MAV, Alberti LR, Ferreira FL, Gomes VM. Historical aspects of } \\
\text { aeromedical transport and aerospace medicine - review. Rev. Med. Minas } \\
\text { Gerais. 2013; } 23 \text { (1): 116-123. [base de dados da Internet]. 2016. Belo Hori- } \\
\text { zonte (MG): [acesso em: } 25 \text { Ago 2018]. Disponível em: rmmg.org/exportar- } \\
\text {-pdf/20/en_v23n1a18.pdf }\end{array}$ \\
\hline & $\begin{array}{l}\text { tical Care Transport. AAOS. Jones and Bartlett Publishers: MA. 2010. 4-19p. } \\
\text { 4- Rajdl E. Air transport: basic and clinical aspects. Revista Médica Clínica }\end{array}$ & $\begin{array}{l}\text { 13- Conselho Federal de Enfermagem. Resolução COFEN 0551/17. [acesso } \\
\text { em } 17 \text { Set 2018]. Disponível em: <http://site.portalcofen.gov.br> }\end{array}$ \\
\hline & $\begin{array}{l}\text { Las Condes. [base de dados na internet]. 2011. [acesso em } 29 \text { Ago 2018]. } \\
\text { 22(3): 389-395. Disponível em:<Www.clc.cl/clcprod/media/contenidos/.../ } \\
\text { 389-396-dr-rajdl.pdf> } \\
\text { 5- Judge, T. (2008). Breathing easier-good news from air medicine. Critical }\end{array}$ & $\begin{array}{l}\text { 14- Santos HGL, Guedes CCP, Aguiar BGC. A segurança do paciente no } \\
\text { transporte aeromédico: uma reflexão para a atuação do enfermeiro. Rev. } \\
\text { Acreditação.2014. [acesso em } 13 \text { Set 2018]. Disponível em: https://dialnet. } \\
\text { unirioja.es/descarga/articulo/5626590.pdf. }\end{array}$ \\
\hline & Care, $12(4), 1-2$ & 15- Raduenz, S. B. D. P., Santos, J. L. G. D., Lazzari, D. D., Nascimer \\
\hline & $\begin{array}{l}\text { 6- da Silva, B. G., Viana, L. L., Faustino, S. D. S. F., Silveira, C. D. P. S., de Car- } \\
\text { valho, V. P., \& de Aguiar Filho, A. S. (2021). Preparação do enfermeiro para }\end{array}$ & $\begin{array}{l}\text { D., Nascimento, K. C. D., \& Moreira, A. R. (2020). Atribuições do enfermeiro } \\
\text { no ambiente aeroespacial. Revista Brasileira de Enfermagem, } 73 \text {. }\end{array}$ \\
\hline & $\begin{array}{l}0 \text { atendimento à múltiplas vítimas no resgate aéreo. Nursing (São Paulo), } \\
24(278), 5948-5957 .\end{array}$ & $\begin{array}{l}\text { 16- Carvalho VP, Pena CD, Pimenta FS, Lopes FL, Souza AAP, Castanheira CH. } \\
\text { Transporte Aeromédico. In: Santana JCB, Melo CL, Dutra BS. Atendimento }\end{array}$ \\
\hline & 7- Ratton JLA. Medicina Intensiva. 4. Ed. Rio de Janeiro: Atheneu; 2005. & Pré-hospitalar: Procedimentos Básicos e Especializados. 2018. Curitiba. CRV. \\
\hline & 8- Dias CP, Chrispim Silva MA, Santos MS, Lopes Ferreira F, Carvalho VP, Alves & \\
\hline & $\begin{array}{l}\text { M. The interdisciplinary team experiences of managing patient safety during } \\
\text { a fixed-wing inter-hospital aeromedical transport: A qualitative study. Int } \\
\text { Emerg Nurs. } 2021 \text { Sep 8;58:101052. }\end{array}$ & $\begin{array}{l}\text { 17- Slaviero RS, Griep R. Perfil epidemiológico dos pacientes atendidos no } \\
\text { ano de } 2014 \text { pelo serviço de transporte aeromédico inter-horpitalar vincu- } \\
\text { lado ao Consórcio Intermunicipal SAMU Oeste, como parte integrante da }\end{array}$ \\
\hline & 9- Vergara, S. C. (2006). Projetos e relatórios de pesquisa. São Paulo: Atlas. & Rede Paraná de Urgência. Revista Thêma et Scientia.2015. 05 (2E).53-62p. \\
\hline & $\begin{array}{l}\text { 10- Bonuzzi, K. L., Muniz-Silva, C. C. S., Santos, O. P., Moraes-Filho, I. M., } \\
\text { Lopes, V. C., \& Silva, R. M. Nurses' performance in air pre-hospital care for }\end{array}$ & \\
\hline
\end{tabular}

